\title{
A cross-sectional study of immune seroconversion to SARS-CoV-2 in frontline maternity health professionals
}

\author{
S. Bampoe, ${ }^{1,2}$ (1) D. N. Lucas, ${ }^{3}$ G. Neall, ${ }^{4}$ P. Sceales, ${ }^{4}$ R. Aggarwal, ${ }^{4}$ K. Caulfield, ${ }^{4}$ \\ D. Siassakos ${ }^{2,5}$ (D) and P. M. Odor ${ }^{1,2}$
}

1 Honorary Associate Professor, Centre for Anaesthesia and Peri-operative Medicine, University College London, UK 2 Consultant, 4 Specialty Registrar, University College London Hospitals NHS Foundation Trust, London, UK

3 Consultant, Northwick Park Hospital, London, UK

5 Associate Professor, University College London, UK

\begin{abstract}
Summary
COVID-19, the respiratory disease caused by SARS-CoV-2, is thought to cause a milder illness in pregnancy with a greater proportion of asymptomatic carriers. This has important implications for the risk of patient-to-staff, staff-to-staff and staff-to-patient transmission among health professionals in maternity units. The aim of this study was to investigate the prevalence of previously undiagnosed SARS-CoV-2 infection in health professionals from two tertiary-level maternity units in London, UK, and to determine associations between healthcare workers' characteristics, reported symptoms and serological evidence of prior SARS-CoV-2 infection. In total, 200 anaesthetists, midwives and obstetricians, with no previously confirmed diagnosis of COVID-19, were tested for immune seroconversion using laboratory IgG assays. Comprehensive symptom and medical histories were also collected. Five out of $40(12.5 \%$; $95 \% \mathrm{Cl} 4.2-$ $26.8 \%)$ anaesthetists, $7 / 52$ (13.5\%; 95\% Cl 5.6-25.8\%) obstetricians and 17/108 (15.7\%; 95\% Cl 9.5-24.0\%) midwives were seropositive, with an overall total of $29 / 200(14.5 \%$; $95 \% \mathrm{Cl} 9.9-20.1 \%)$ of maternity healthcare workers testing positive for IgG antibodies against SARS-CoV-2. Of those who had seroconverted, 10/29 (35.5\%) were completely asymptomatic. Fever or cough were only present in 6/29 (21\%) and 10/29 (35\%) respectively. Anosmia was the most common symptom occurring in 15/29 (52\%) seropositive participants and was the only symptom that was predictive of positive seroconversion (OR $18 ; 95 \% \mathrm{Cl} 6-55)$. Of those who were seropositive, $59 \%$ had not self-isolated at any point and continued to provide patient care in the hospital setting. This is the largest study of baseline immune seroconversion in maternity healthcare workers conducted to date and reveals that one out of six were seropositive, of whom one out of three were asymptomatic. This has significant implications for the risk of occupational transmission of SARS-CoV-2 for both staff and patients in maternity units. Regular testing of staff, including asymptomatic staff should be considered to reduce transmission risk.
\end{abstract}

Correspondence to: S. Bampoe

Email: Sohail.Bampoe@NHS.net

Accepted: 22 July 2020

Keywords: COVID-19; obstetrics; seroconversion

Twitter: @Drsambam; @noolslucas; @DSiassakos; @peteodor

\section{Introduction}

Serological testing to assess the extent of seroconversion in a population plays a vital role in containing an infectious outbreak. It can provide information on the prevalence of the infection in a cohort of people and also provide information regarding how a disease spreads. In the UK, 
current data from the Office of National Statistics suggest that in May 2020, 6.78\% of the population in England had detectable antibodies to SARS-CoV-2, the virus that causes COVID-19 [1]. A similar study of community seroconversion in Los Angeles, USA, reported a comparable seroconversion proportion of $4 \%$ [2].

A particular area of concern is people who seroconvert without having experienced clinical features of infection. A study of 215 pregnant women presenting for delivery in New York, USA, reported that $13.7 \%$ tested positive for active SARS-CoV-2 infection, $87.9 \%$ of whom were asymptomatic [3]. Although this study did not test for seroconversion, these data might suggest that the overall infection rate in the pregnant population may be much higher than the general population due to the presence of a higher proportion of asymptomatic carriers.

Obstetric care has, by necessity, continued throughout the COVID-19 pandemic. Given that third-trimester pregnancy care involves multiple ongoing encounters with healthcare professionals and a potentially high proportion of reported asymptomatic carriers, obstetric healthcare professionals may be at an elevated risk of patient-to-staff, staff-to-staff and staff-to-patient transmission. Several studies have reported an elevated risk of transmission and seroconversion in frontline non-obstetric healthcare workers in several settings, including Wuhan, China and London, UK $[4,5]$. Only one single-centre study reports seroconversion rates in an obstetric unit; however, this was following a COVID-19 outbreak among unit staff in that particular unit in Ohio, USA [6]. To date, no studies have reported a baseline seroconversion rate of obstetric healthcare workers providing frontline, patient-facing care during the current SARS-CoV-2 pandemic.

The primary aim of this study was to investigate the prevalence of previously undiagnosed SARS-CoV-2 infection in two tertiary level maternity units in London, UK. The secondary aim was to determine associations between healthcare worker characteristics, reported symptoms and serological evidence of prior SARS-CoV-2 infection.

\section{Methods}

Following approval from the NHS Health Research Authority, we recruited maternity healthcare workers from two large central London teaching hospitals between 11 May 2020 and 5 June 2020. Inclusion criteria were midwives, anaesthetists or obstetricians working on labour ward or post-natal care, aged $\geq 18$ years. Participants were not included if they had a history of SARS-Cov-2 infection diagnosed by polymerase chain reaction from nasopharyngeal swabs, or if they were currently symptomatic for SARS-CoV-2 infection. Staff working in nonpatient facing roles were also not included. Participation was voluntary and informed written consent was taken from all participants.

Participants completed a questionnaire that captured baseline characteristics, self-reported medical history and symptom history since January 2020. After completion of the questionnaire, the research team collected $10 \mathrm{ml}$ of blood in serum separating tubes from each participant. Blood samples were centrifuged for $10 \mathrm{~min}$ at $3100 \mathrm{rpm}$ and separated serum was frozen at $-80^{\circ} \mathrm{C}$ ahead of transport to the laboratory.

Serological testing was performed using Abbott Laboratories' (Chicago, IL, USA) two-step, chemiluminescent micro-particle immunoassay technology, approved by the United States Food and Drug Administration and Public Health England. This assay detects the presence of lgG antibodies formed against the nucleocapsid protein antigen of SARS-CoV-2 in samples of patient serum [7]. Serum samples are combined with antigen-coated paramagnetic micro-particles and assay diluent before incubation. After a wash cycle, anti-human IgG acridinium-labelled conjugate is added and a further wash cycle is performed before the addition of trigger solutions. As a result of the addition of the trigger solutions, a chemiluminescent reaction occurs that is measured as a relative light unit. The relative light unit is measured by Abbott Laboratories' proprietary ARCHITECT isystem. The relative light units detected by the ARCHITECT system optics are directly related to the amount of IgG antibodies present [7]. The system also measures the relative light units from a control sample. If dividing the sample relative light units by the control relative light units produces an index of $<1.4$, the sample is considered to be negative for SARSCoV-2 lgG antibodies. An index $>1.4$ is considered to be a positive result [7]. This test has $100 \%$ sensitivity and $99.6 \%$ specificity as reported by the manufacturer and United States Food and Drug Administration [8].

All data collected through the questionnaire, in addition to results of serology testing, were entered into a RedCap $^{\mathrm{TM}}$ (Vanderbilt University, Nashville, TN, USA) relational database system. The required sample size was estimated by assuming a prevalence of seroconversion of $15 \%$. Enrolling a sample size of 196 participants would allow precision of $\pm 5 \%$ with confidence intervals of $95 \%$. If the actual prevalence was lower than estimated, then the precision of our data sample would improve.

Statistical analysis was performed using SPSS (IBM, Armonk, NY, USA). The proportion of seroconversion was calculated with binomial $95 \% \mathrm{Cl}$. Categorical variables were 
analysed using Chi-square or Fisher's exact test. Binary logistic regression analysis was performed to determine factors associated with seroconversion, including age, sex, $\mathrm{BMI}$, occupational role, ethnicity and the presence of recognised COVID-19 symptoms. The model's calibration, or its fit to the data, was subsequently assessed with the Hosmer-Lemeshow test; this was determined by the degree of agreement between the risk score probabilities that had been predicted by the model, and the probabilities that were actually observed. Interaction effects were introduced to optimise model fit.

\section{Results}

A total of 200 obstetric healthcare professionals were tested over a 3-week period between May and June 2020. This included 40 anaesthetists practising in maternity, 108 midwives and 52 obstetricians. Baseline characteristics are reported in Table 1.

IgG antibodies to SARS-CoV-2 were detected in $29 / 200 \quad(14.5 \% ; \quad 95 \% \mathrm{Cl} \quad 9.9-20.1 \%)$ of obstetric healthcare workers. Seroconversion among individual staff groups was: midwifery $17 / 108(15.7 \%$; $95 \% \mathrm{Cl} 9.5-$ $24.0 \%)$; anaesthesia $5 / 40(12.5 \% ; 95 \% \mathrm{Cl} \quad 4.2-26.8 \%)$; obstetrics $\quad 7 / 52 \quad(13.5 \% ; \quad 95 \% \mathrm{Cl} \quad 5.6-25.8 \%)$. Seroconversion proportions were similar at both hospitals. Of the seroconverted staff members, 10/29 (35\%) were completely asymptomatic. In those with a history of symptoms and who seroconverted, the most common symptoms were anosmia 15/29 (52\%) and headache. Of the seropositive participants, 10/29 (35\%) reported a history of dry or productive cough and 6/29 (21\%) reported a history of fever (Table 2 ).

Twelve out of 29 (41\%) seropositive participants had self-isolated at least once during the pandemic. Of these, 17 (59\%) had continued to work throughout the period, either because they were asymptomatic or their symptoms did not qualify them at the time for selfisolation (10 and 7 members of staff respectively). Logistic regression found that the presence of anosmia was the only significant predictor of antibody seroconversion (OR 18; 95\% Cl 6-55). No other baseline chararcteristics or symptom variables were predictive of immune seroconversion including age, staff group, BMI, ethnicity, fever or cough (Table 3).

\section{Discussion}

This is the largest study to report baseline immune seroconversion to SARS-CoV-2 in obstetric healthcare workers to date. We found that one of every six frontline, obstetric healthcare workers had been infected with SARS-
Table 1 Participant baseline, occupational and comorbid characteristics. Values are median (IQR [range]) or number (proportion).

\begin{tabular}{|c|c|}
\hline & Participants $(n=200)$ \\
\hline Age; years & $37(30-37[21-66])$ \\
\hline Weight; kg & $70(60-80[44-118])$ \\
\hline $\mathrm{BMl} ; \mathrm{kg} \mathrm{m}^{-2}$ & $24.2(21.8-27.7[16.7-40.2])$ \\
\hline Female & $167(84 \%)$ \\
\hline \multicolumn{2}{|l|}{ Occupation } \\
\hline Anaesthetist & $40(20 \%)$ \\
\hline Midwife & $108(54 \%)$ \\
\hline Obstetrician & $52(26 \%)$ \\
\hline \multicolumn{2}{|l|}{ Ethnicity } \\
\hline Caucasian & $139(70 \%)$ \\
\hline BAME total & $61(31 \%)$ \\
\hline Asian or Asian British & $25(13 \%)$ \\
\hline Black or Black British & $25(13 \%)$ \\
\hline Mixed & $9(5 \%)$ \\
\hline Other & $2(1 \%)$ \\
\hline \multicolumn{2}{|l|}{ Smoke } \\
\hline Never smoked & $141(71 \%)$ \\
\hline Former smoker & $36(18 \%)$ \\
\hline Smoker & $13(7 \%)$ \\
\hline Unknown & $10(5 \%)$ \\
\hline \multicolumn{2}{|l|}{ Comorbidities } \\
\hline $\begin{array}{l}\text { Immunosuppressed/ } \\
\text { receiving steroids }\end{array}$ & 0 \\
\hline Cardiac disease & 0 \\
\hline Hypertension & $10(5 \%)$ \\
\hline $\begin{array}{l}\text { Chronic obstructive } \\
\text { pulmonary disorder }\end{array}$ & 0 \\
\hline Diabetes & $1(0.5 \%)$ \\
\hline $\begin{array}{l}\text { Diabetes with end organ } \\
\text { complications }\end{array}$ & 0 \\
\hline Asthma & $21(11 \%)$ \\
\hline Chronic kidney disease & 0 \\
\hline $\begin{array}{l}\text { Moderate/severe } \\
\text { hepatic disease }\end{array}$ & $1(0.5 \%)$ \\
\hline
\end{tabular}

CoV-2 as revealed by the presence of $\lg G$ antibodies. Seroconversion was more than twice as prevalent in UK obstetric healthcare workers as in the general population of the UK and three times as prevalent as in the general population of the USA [1, 2]. However, the prevalence of seroconversion of healthcare workers in these London hospitals was similar to estimates of seroconversion in the general population in Greater London at $14.5 \%$, based on sampling from blood donors [9].

Although these results would suggest that obstetric healthcare workers are at a similar risk of exposure to 
Table 2 Reported symptoms and SARS-CoV-2 detection by serology, values are number (proportion).

\begin{tabular}{llll} 
Symptoms & $\begin{array}{l}\text { Seroconverted } \\
\mathbf{n}=\mathbf{2 9}\end{array}$ & $\begin{array}{l}\text { No antibodies detected } \\
\mathbf{n = 1 7 1}\end{array}$ & p value \\
\hline Asymptomatic & $10(35 \%)$ & $59(35 \%)$ & 0.998 \\
\hline Dry cough or fever or anosmia & $16(55 \%)$ & $53(31 \%)$ & 0.113 \\
\hline Dry cough & $10(35 \%)$ & $41(24 \%)$ & 0.230 \\
\hline Fever & $6(21 \%)$ & $15(9 \%)$ & 0.053 \\
\hline Anosmia & $15(52 \%)$ & $9(5 \%)$ & $<0.001$ \\
\hline Headache & $14(48 \%)$ & $61(36 \%)$ & 0.195 \\
\hline Myalgia & $12(41 \%)$ & $42(25 \%)$ & 0.593 \\
\hline Nasal congestion & $6(21 \%)$ & $25(15 \%)$ & 0.404 \\
\hline Sore throat & $6(21 \%)$ & $52(30 \%)$ & 0.286 \\
\hline Shortness of breath & $5(17 \%)$ & $24(14 \%)$ & 0.650 \\
\hline Diarrhoea & $4(14 \%)$ & $24(14 \%)$ & 0.972 \\
\hline Nausea or vomiting & $3(10 \%)$ & $14(8 \%)$ & 0.700 \\
\hline Photophobia & $2(7 \%)$ & 0 & 0.020 \\
\hline Productive cough & $1(3 \%)$ & $9(5 \%)$ & 0.678 \\
\hline Abdominal pain & $1(3 \%)$ & $12(7 \%)$ & 0.471 \\
\hline
\end{tabular}

Table 3 Factors associated with detection of antibodies to SARS-CoV-2 in maternity healthcare workers. Values are OR (95\% CI).

\begin{tabular}{|llr|}
\hline $\begin{array}{l}\text { Characteristic } \\
\text { Age; years }\end{array}$ & OR $(\mathbf{9 5} \mathbf{9} \mathbf{C l})$ & \\
\hline $18-30$ & & 0.921 \\
\hline $31-50$ & $1.1(0.3-4.0)$ & 0.872 \\
\hline $51-70$ & $1.1(0.2-5.4)$ & 0.494 \\
\hline BMI $>30$ & $0.6(0.1-2.6)$ & \\
\hline Sex & & \\
\hline Female & $1.0($ ref) & \\
\hline Male & $1.1(0.2-5.5)$ & 0.883 \\
\hline Ethnicity & & 0.362 \\
\hline Caucasian & $1.0($ ref) & 0.611 \\
\hline BAME & $1.6(0.6-4.5)$ & 0.001 \\
\hline Fever & $1.5(0.3-7.7)$ & 0.858 \\
\hline Anosmia & $18.2(6.0-55.2)$ & 0.281 \\
\hline Headache & $1.1(0.4-3.4)$ & 0.864 \\
\hline Self-isolated & $0.4(0.1-1.9)$ & \\
\hline Dry cough & $1.0(0.3-3.4)$ & \\
\hline
\end{tabular}

COVID-19 as the general population, the prevalence of seroconversion among this staff group appears to be lower than reported in other frontline healthcare worker groups. Houlihan et al. measured seroconversion in 200 healthcare workers in London working in the emergency department, acute medical unit, COVID-19 cohort wards, ICU and haematology wards. Using serial serology tests over three months, they reported a collective seroconversion proportion of $45.3 \%$ [5]

One possible explanation for the difference in seroconversion rates between obstetric healthcare workers and other frontline healthcare workers could be due to the transmission characteristics of SARS-CoV-2. The transmission characteristics of SARS-CoV-2 appear to be similar to those of influenza in that viral shedding appears to occur in those who are asymptomatic and also in the presymptomatic phase of infection [10]. In patients infected with influenza, quantitative viral load in asymptomatic individuals is less in upper respiratory tract secretions and the duration of viral shedding is also shorter than in symptomatic individuals [11]. Therefore, if the above assumptions are correct, then it may be reasonable to extrapolate that asymptomatic individuals infected with SARS-CoV-2 pose a lower transmission risk to others. Hence the reported greater proportion of asymptomatic infection in pregnancy and generally milder symptom profile in pregnant patients may explain why obstetric healthcare workers appear to have a lower risk of occupational viral transmission, as SARS-CoV-2 pregnant patients may be less infective than respiratory or acute emergency patients $[3,12]$.

The risk posed by infectious staff members to colleagues, pregnant women and their babies is unknown. At the beginning of the current SARS-CoV 2 pandemic, the UK government advised all those with a persistent cough or fever $>37.8^{\circ} \mathrm{C}$ to self-isolate. Our data revealed that only $41 \%$ of healthcare workers who were seropositive met those 
criteria and self-isolated at any point. This means that $59 \%$ continued to work and commute despite active SARS-CoV-2 infection. Indeed, our data showed that in this population, neither cough nor fever predicted seropositivity and the only symptom predictive of immune seroconversion was anosmia. The UK Government has since added anosmia as a symptom that mandates self-isolation. Our study strongly supports this updated advice, albeit it would still fail to isolate approximately $6 / 10$ infected staff members. Of importance, it was not until 15 June 2020 that all staff members in patient-facing areas were advised by Public Health England to wear surgical masks to reduce the risk of infection to others [13].

In maternity settings, SARS-CoV-2 poses risks to staff and women alike, but also potentially to their babies. The UK Obstetric Surveillance System collected data from 427 pregnant women admitted with confirmed SARS-CoV-2 infection and reported an estimated incidence of maternal hospitalisation with COVID-19 of 4.9 per 1000 pregnancies [12]. The majority of patients were in the second and third trimesters of pregnancy and suffered mild disease. In this study, of 266 completed maternities, there were three stillbirths, with two potentially related to COVID-19. With the current UK stillbirth rates about 4 in 1000, only one stillbirth would have been expected in the UK Obstetric Surveillance System cohort $[13,14]$. Studies of placentas from COVID-19 infected women have highlighted the potential of the wider adverse impact of COVID-19 infection on pregnancy [15]. Relative to controls, placentas of women with COVID-19 show increased prevalence of decidual arteriopathy and other features of maternal vascular malperfusion, a pattern of placental injury reflecting abnormalities in oxygenation within the inter-villous space, associated with adverse perinatal outcomes [15]. Moreover, even if the incidence of serious neonatal COVID-19 infection is low, possible vertical or perinatal transmission has been reported and the issue of separation of babies from infected mothers has been debated $[16,17]$. Transmission to the newborn or to their mothers by infectious asymptomatic members of staff present at birth has not been investigated to date. Knowledge of the incidence of symptomatic and asymptomatic seroconversion of maternity staff and how it compares with the general population, with staff in other healthcare settings and with maternity patients, is useful for providing context to such investigations, particularly in case of future additional waves of infection.

Until we have robust evidence as to the risk posed by asymptomatic infected individuals to others, and as to the risk of COVID-19 to babies, particularly in utero, our study suggests that extreme caution is advisable in maternity settings, particularly the consistent use of effective personal protective equipment. We also recommend that all obstetric healthcare institutions should consider regular serology testing for staff, as well as the immediate isolation of any staff who develop anosmia, even in the absence of cough or fever. Regular testing and consistent use of personal protective equipment and hand-washing are likely to be the cornerstones of pandemic control.

Our study does have limitations. We used antibody seroconversion as a marker of previous COVID-19 infection. Based on measurements in other similar viruses [18], the duration of antibody titres sufficient to be detected is likely to be for at least 6 months. However, the exact duration of SARS-CoV-2 lgG antibody persistence remains unknown and recent studies have shown that mild cases might end up seronegative and only exhibit mucosal IgA [Cervia et al., unpublished observations, doi.org/10.1101/ 2020.05.21.108308]. As such it is possible that some individuals who were infected earlier in the year, as well as some mild cases, did not exhibit sufficient antibody response at the date of testing. Our testing methodology may therefore underestimate the true seroconversion prevalence.

\section{Acknowledgements}

This study was funded by a grant from the University College London Hospitals Charity. SB is supported by an award from the National Institute for Health Research UCL/ UCLH Biomedical Research Centre, London, UK. No other external funding or competing interests declared.

\section{References}

1. Office for National Statistics. Coronavirus (COVID-19) Infection Survey Coronavirus (COVID-19) Infection Survey pilot: 28 May 2020. https://www.ons.gov.uk/peoplepopulationandcommunity/ healthandsocialcare/conditionsanddiseases/bulletins/corona viruscovid19infectionsurveypilot/28may2020 (accessed 01/06/ 2020).

2. Sood N, Simon P, Ebner P, et al. Seroprevalence of SARS-CoV-2Specific antibodies among adults in Los Angeles County, California, on April 10-11, 2020. Journal of the American Medical Association 2020; 323: 2425-7.

3. Sutton D, Fuchs K, D'Alton M, Goffman D. Universal screening for SARS-CoV-2 in women admitted for delivery. New England Journal of Medicine 2020; 382: 2163-4.

4. Pan A, Liu L, Wang C, et al. Association of public health interventions with the epidemiology of the COVID-19 outbreak in Wuhan, China. Journal of the American Medical Association 2020; 323: 1915-23.

5. Houlihan C, Vora N, Byrne T, et al. Pandemic peak SARS-CoV-2 infection and seroconversion rates in London frontline healthcare workers. Lancet 2020. Epub 9 July. https://doi.org/10. 1016/S0140-6736(20)31484-7.

6. Kiefer MK, McKiever ME, Russo JR, et al. Exposure and seroconversion to SARS-CoV-2 among obstetric healthcare providers following a contained outbreak. American Journal of 
Obstetrics and Gynecology 2020. Epub 15 June. https://doi. org/10.1016/j.ajog.2020.06.029.

7. Public Health England. Evaluation of the Abbott SARS-CoV-2 IgG for the detection of anti-SARS- CoV-2 antibodies. 2020. https://assets.publishing.service.gov.uk/government/uploads/ system/uploads/attachment_data/file/890566/Evaluation_of_ Abbott_SARS_CoV_2_IgG_PHE.pdf(accessed 23/07/2020).

8. US Food and Drug Administration. EUA Authorized Serology Test Performance. 2020. https://www.fda.gov/medical-device s/emergency-situations-medical-devices/eua-authorized-se rology-test-performance (accessed 10/06/2020).

9. Weekly Coronavirus Disease 2019 (COVID-19) Surveillance Report. Week 24. 2020. https://assets.publishing.service.gov. uk/government/uploads/system/uploads/attachment_data/file/ 891721/Weekly_COVID19_Surveillance_Report_-_week_24.pdf (accessed 20/06/2020).

10. Gandhi M, Yokoe DS, Havlir DV. Asymptomatic transmission, the Achilles' heel of current strategies to control Covid-19. New England Journal of Medicine 2020; 382: 2158-60.

11. Ip DKM, Lau LLH, Leung NHL, et al. Viral shedding and transmission potential of asymptomatic and paucisymptomatic influenza virus infections in the community. Clinical Infectious Diseases 2017; 64: 736-42.

12. Knight $M$, Bunch $K$, Vousden $N$, et al. Characteristics and outcomes of pregnant women admitted to hospital with confirmed SARS-CoV-2 infection in UK: national population based cohort study. British Medical Journal 2020; 369: m2107.

13. Office for National Statistics. Births in England and Wales: 2018. 2019. https://www.ons.gov.uk/peoplepopulationandc ommunity/birthsdeathsandmarriages/livebirths/bulletins/ birthsummarytablesenglandandwales/2018 (accessed 18/06/2020)

14. MBRRACE-UK. Perinatal Mortality Surveillance Report UK Perinatal Deaths for Births from January to December 2016. 2018. https://www.npeu.ox.ac.uk/downloads/files/mbrrace-uk/ reports/MBRRACE-UK\%20Perinatal\%20Surveillance\%20Summa ry\%20Report\%20for\%202016\%20-\%20June\%202018.pdf (accessed 18/06/2020).

15. Shanes ED, Mithal LB, Otero S, Azad HA, Miller ES, Goldstein JA. Placental pathology in COVID-19. American Journal of Clinical Pathology 2020; 154: 23-32.

16. Dong L, Tian J, He S, et al. Possible vertical transmission of SARS-CoV-2 from an infected mother to her newborn. Journal of the American Medical Association 2020; 323: 1846-8.

17. Egloff C, Vauloup-Fellous $C$, Picone $O$, Mandelbrot L, Roques $P$. Evidence and possible mechanisms of rare maternal-fetal transmission of SARS-CoV-2. Journal of Clinical Virology 2020; 128: 104447.

18. Tang F, Quan Y, Xin ZT, et al. Lack of peripheral memory B call responses in recovered patients with severe acute respiratory syndrome: a six year follow up study. Journal of Immunology 2011; 186: 7264-8. 